\title{
Le 11 septembre 1942 : Les cheminots et les déportés juifs en gare de Fives-Lille
}

Daniel Mandelbaum et Jacqueline Mandelbaum-Bleibtreu

\section{(2) OpenEdition}

1 Journals

Édition électronique

URL : https://journals.openedition.org/tsafon/1471

DOI : $10.4000 /$ tsafon. 1471

ISSN : 2609-6420

Éditeur

Association Jean-Marie Delmaire

Édition imprimée

Date de publication : 1 décembre 2018

Pagination : 169-176

ISSN : 1149-6630

\section{Référence électronique}

Daniel Mandelbaum et Jacqueline Mandelbaum-Bleibtreu, «Le 11 septembre 1942 : Les cheminots et les déportés juifs en gare de Fives-Lille », Tsafon [En ligne], 76 | 2018, mis en ligne le 30 juin 2019, consulté le 24 juin 2021. URL : http://journals.openedition.org/tsafon/1471 ; DOI : https://doi.org/ $10.4000 /$ tsafon. 1471 


\title{
Édition
}

\section{Le 11 septembre 1942 \\ Les cheminots et les déportés juifs en gare de Fives-Lille}

\author{
Daniel Mandelbaum \\ Jacqueline Mandelbaum-Bleibtreu
}

Paris le 20 septembre 2018

Le témoignage qui suit est basé sur les récits de nos parents, Tauba Mandelbaum née Weissblum et Lejbus (Léon) Mandelbaum, concernant la journée du 11 septembre 1942. Cette journée s'inscrit dans notre histoire familiale comme une pierre à deux faces, l'une noire, l'autre blanche.

Le 11 septembre 1942, jour de Rosh Hashana, nos parents sont réveillés, vers 5 h30 du matin, par des coups frappés à la porte de leur maison, 6 rue du Marais à Lens. Un gendarme français et un feldgendarme allemand armés leur enjoignent de se préparer rapidement et de les suivre jusqu'à la gare SNCF où ils prendront, avec les autres juifs de la ville, un train pour un camp de travail à l'Est. À Tauba qui demande s'il faut prendre des vêtements chauds, il est répondu qu'ils n'ont droit qu'à deux bagages légers et que surtout il faut privilégier tout ce qu'ils ont de peu encombrant et précieux, argent et bijoux. Il est clair qu'il faut faire très vite, le ton est impérieux, agacé, sans brutalité excessive. 
Tauba gardera de leur traversée de Lens à pied, escortés par la «maréchaussée» et rejoints par d'autres familles juives de la communauté lensoise, un sentiment d'humiliation et de colère qui ne s'effacera jamais : les rideaux des maisons voisines soulevés pour voir et vite relâchés pour ne pas être vus, les regards indifférents, les quolibets, les démonstrations de satisfaction d'être débarrassés des juifs et aucune manifestation de sympathie.

À la gare se trouvent réunies plus de 200 personnes de la ville : jeunes, vieillards, malades, invalides, bébés qui attendent les juifs venant des localités voisines. Dès le début de la rafle, Tauba n'a qu'une idée en tête, s'enfuir. Militants communistes, d'abord en Pologne pour Lejbus puis à Anvers et Paris, ils n'ignorent rien de ce que sont les nazis et leur antisémitisme fanatique et ils voudraient se battre contre cette barbarie. Tauba sent que lorsqu'on envoie des individus en camp de travail, on ne prend aucune bouche inutile. Il ne peut donc y avoir qu'une raison à cette déportation : leur élimination. L'attente est longue. Lorsque le train est enfin au complet, en début d'après-midi, on ne leur a rien donné à boire ni à manger depuis leur arrestation. Les policiers français les rembarrent brutalement lorsqu'ils réclament, s'attirant même, un comble, la désapprobation d'un jeune soldat allemand.

Tauba et Lejbus se retrouvent dans un compartiment avec une petite fille de neuf ans et ses parents, un couple et leur nourrisson. À l'entrée en gare de Fives-Lille, le bébé pleure car il fait chaud; sa mère n'a plus de lait et personne n'a d'eau. Le train s'arrête : sur le quai des soldats allemands armés gardent le convoi. Tauba qui continue à chercher un moyen de fuir et sait qu'elle ne le trouvera pas à l'intérieur du compartiment, décide de descendre trouver quelque chose à boire. La voilà sur le quai. La sentinelle braque son fusil vers elle et lui enjoint de remonter dans le train. En allemand, qu'elle parle bien, Tauba explique qu'elle veut aller au buffet de la gare pour satisfaire une envie pressante et trouver de l'eau pour le bébé du compartiment qui n'a rien bu depuis des heures. Le soldat, jeune, lui répond qu'elle peut faire ses besoins sur le quai. "Wir sind doch keine Hunde und noch Menschen» "Nous sommes encore des êtres humains et pas des chiens » réplique-t-elle, indignée. Est-ce parce qu'elle parle bien allemand, parce qu'elle est très blonde aux yeux bleus, parce qu'il est désarçonné... il a un réflexe normal dans cette folie, il appelle un cheminot qui se trouve à proximité et lui demande d'accompagner notre mère au buffet de la gare et de la ramener à son wagon. 
Pendant le trajet, le cheminot semble bouleversé. "Pourquoi pleurez-vous » demande Tauba? « Parce qu'on ne peut pas voir ça sans être remué ». "Pleurer ne sert à rien, il faut agir ». " Maintenant et ici c'est impossible, mais si vous voulez vous enfuir, je peux vous aider ». Il lui explique que leur train va bientôt être dirigé vers la gare de triage où les juifs doivent rejoindre un autre convoi. Durant ce court trajet au ralenti, le train va emprunter une courbe et longer le mur d'enceinte dont il sera séparé par un autre train en stationnement pour réparation. Cette partie n'est pas gardée, donc aveugle. Tauba n'aurait qu'à ouvrir la porte donnant à contre-voie, sauter en marche, passer sous le train arrêté et emprunter l'échelle qu'il aura appuyée contre le mur. Parvenue à son sommet, il lui restera à sauter dans la rue où il l'attendra.

Tauba accepte, sans l'ombre d'une hésitation, cette main tendue et remonte dans la voiture avec de l'eau. Elle explique aux autres passagers ce projet d'évasion et demande qui veut le partager. Personne n'est tenté et tous essaient de la dissuader, sauf Lejbus, bien sûr : " Nous partons travailler, ce sera dur mais on peut s'en sortir. Si nous tentons de nous enfuir maintenant, les soldats allemands nous fusilleront ! ». Mais il n'y a aucun doute dans l'esprit de nos parents sur l'avenir qui les attend tous au bout du voyage... Il faut saisir la chance qui se présente. «Mieux vaut mourir debout que vivre à genoux! » répond Tauba, paraphrasant la Pasionaria, Dolores Ibarruri, dans un discours au Vel d'Hiv le 8 septembre 1936, lors d'un meeting de soutien à la République espagnole.

Nos parents élaborent alors une stratégie «de combat». Tauba parle le français avec juste un soupçon d'accent, qui peut aisément passer pour celui de l'Est de la France. Elle partira la première. Si elle échoue, Lejbus, d'une manière ou d'une autre, parviendra peut-être à survivre à cette guerre. Le couple espère minimiser ainsi les risques que leur fils Daniel, 2 ans et demi, caché au préventorium de l'abbaye de Valloires dans la Somme, se retrouve sans parents. Si Tauba réussit, le cheminot essaiera de prévenir Lejbus.

Le train redémarre. Le bébé désaltéré a cessé de pleurer. On arrive effectivement dans une courbe, on ralentit, tout correspond à la description du cheminot. Tauba ouvre la porte donnant sur la voie. Au dernier moment, la mère de la petite fille pousse son enfant : "Madame, je suis d'accord avec vous mais je n'ai pas le courage de vous suivre. Prenez ma fille avec vous! ». Toutes deux sautent, courent jusqu'à la rame en stationnement, passent sous les wagons et atteignent le mur 
d'enceinte. L'échelle est bien là. Pas de gardes dans cette partie de la gare, le train arrêté cache la femme et la fillette qui grimpent rapidement. De l'autre côté, le cheminot les attend. Il récupère l'enfant, Tauba saute et se reçoit mal. Sa cheville est foulée, très douloureuse. Le cheminot modifie ses plans et conduit les fugitives vers une maison située juste en face. Une femme, qu'il semble bien connaître, accepte d'accueillir les évadées pour les mettre à l'abri. Le cheminot retourne rapidement à la gare. Il ne faut pas que son absence se prolonge et soit remarquée. Il va tenter maintenant de prévenir Lejbus du succès de «l'opération ».

Entre temps, le train est arrivé à la gare de triage où doit s'effectuer le transfert «des passagers » dans un autre convoi. Nos parents l'ont toujours décrit comme un train à destination d'Auschwitz. En fait, il partait pour Malines, le Drancy belge. Lejbus, sur le point d'entrer avec d'autres hommes dans un wagon à bestiaux, voit arriver un cheminot qui semble vérifier le train, tapant sur les attelages, les essieux et appelant: «Mandelbaum, Mandelbaum!». Lejbus s'écrie: «C'est moi Mandelbaum ». Le cheminot s'approche : "Votre femme a réussi, elle est en sécurité avec la fillette ». Après les remerciements, Lejbus interroge : « Moi aussi je veux partir ». "Il est trop tard, les Allemands sont devenus méfiants et renforcent les contrôles ». « Ce n'est jamais trop tard; est-ce que vous ne pouvez pas me faire passer pour un de vos collègues? ». Le cheminot acquiesce. Des agents intérimaires ont été recrutés pour cette journée particulière. Ils portent un brassard noir qui leur sert de laisser-passer auprès des sentinelles allemandes. Le cheminot donne à Lejbus un brassard, qu'il avait dans sa poche, un porte-bloc et un crayon pour faire semblant de prendre des notes. "Surtout, vous ne parlez pas ! » lui enjoint-il.

C'est ainsi que tous deux traversent la gare et rejoignent la sortie, non sans se faire interpeller par un soldat allemand, apparemment rassuré par les explications du cheminot présentant Lejbus comme un gars venu les aider en raison de l'affluence. Ils gagnent rapidement la maison voisine où Tauba et Lejbus se retrouvent. Ils sont momentanément sauvés. Le cheminot se présente : il s'appelle Marcel Hoffmann.

La suite de la guerre fut pleine de péripéties pour nos parents qui doivent leur survie à leur courage, à la chance et bien sûr à l'aide de nombreux Français, résistants ou simplement compatissants. Notre mère, grâce au réseau du pasteur Nick, fut cachée, en tant que monitrice, au préventorium de Palente près de Besançon. Elle ne voulait pas quitter la 
France pour pouvoir maintenir un lien, au moins épistolaire, avec leur petit garçon. Notre père, qui était immédiatement trahi par son accent, réussit à passer en Suisse où il fut interné dans un camp de travail. Après la guerre, ils se retrouvèrent et récupérèrent leur fils. Ils pensaient que Marcel Hoffmann avait été arrêté pour fait de résistance et fusillé. Ils lui doivent la vie, leur fils Daniel d'avoir pu retrouver sa famille et leur fille Jacqueline d'avoir pu naître en 1946.

Cette histoire familiale s'est gravée dans nos mémoires d'enfant et nous l'avons transcrite telle qu'elle nous a été racontée, limitée, nous en sommes bien conscients, par ce que nos parents ont voulu ou pu nous transmettre. Notre émotion fut donc très grande, lorsqu'il a été possible de confronter ce récit aux récents travaux des historiens, de constater qu'il s'emboîtait parfaitement dans le puzzle de l'histoire factuelle et globale. Certes, nos parents n'avaient aucune idée de l'action concertée des cheminots de Fives-Lille et de la population locale et ont pensé à un acte isolé et héroïque de Marcel Hoffmann. Les détails précis que nous avons découverts récemment nous expliquent et complètent le récit de nos parents. Marcel Hoffmann n'a pas été convaincu par notre mère, il tentait, comme d'autres cheminots, depuis le matin de soustraire à la déportation, des juifs, le plus souvent réticents et effrayés par sa démarche. Notre père l'a vu frapper des éléments du train avec un outil car il était «visiteur du matériel », ce qui lui permettait de circuler à sa guise à travers la gare et de s'approcher des trains. Les photos de la gare de triage corroborent point par point ce qui nous a été raconté, etc...

Beaucoup de questions restent cependant en suspens et nous taraudent: pourquoi nos parents ont-ils cru Marcel Hoffmann mort pendant la guerre? Qu'est devenue la petite fille dont nous ne connaissons pas le nom et que Tauba a soustraite à la déportation? D'après nos parents, elle aurait passé le reste de la guerre dans la famille qui a ouvert sa porte ce 11 septembre 1942 et après la guerre aurait retrouvé une tante et émigré en Israël. Pourquoi nos parents ne nous ontils jamais emmenés sur les lieux même de cette histoire ? Grâce à la journée de commémoration organisée l'an dernier à la gare de FivesLille, nous avons rencontré la fille, le petit-fils et l'arrière-petite-fille de Marcel Hoffmann et pu leur exprimer notre profonde gratitude. 


\section{Remarques de la rédaction}

- Des témoignages de la part de juifs raflés à Lens, revenus de la déportation, confirment la présence de Lensois, sur leur parcours vers la gare, qui restent insensibles au spectacle de ce lamentable défilé, voire manifestent une certaine satisfaction. En revanche, Frieda Thau, arrêtée ce 11 septembre 1942 à Lens, se souvient que sa patronne, elle était couturière, s'est déplacée jusqu'à la gare pour lui apporter des chocolats, geste dérisoire mais très réconfortant pour la jeune fille de 18 ans. Frieda a rappelé ce geste généreux, en diverses occasions. Lire entre autres, son bref témoignage sur le site des Anonymes, Justes, Persécutés durant la période nazie ajpn.org.

- Le réseau du pasteur Nick:

Le pasteur Henri Nick s'est établi, à la fin du XIX ${ }^{\mathrm{e}}$ siècle, dans la paroisse très populaire de Fives, de la proche banlieue de Lille. Il eut une très grande influence sur ses paroissiens en prenant la défense des ouvriers durement exploités et en luttant contre l'alcoolisme et la prostitution. Il organisa des colonies de vacances sur la côte d'Opale pour les enfants d'ouvriers, il protégea également des objecteurs de conscience durant les années 1930. Pendant la Seconde Guerre mondiale, il se dépensa pour cacher les proscrits et particulièrement les juifs. Sa paroisse jouxtant la gare de Fives-Lille, les quelques habitants, qui avaient récupéré des juifs sortis de la gare grâce aux cheminots, se sont tournés vers lui pour les cacher. C'est alors que se mit en place un réseau autour du pasteur Nick pour disperser et cacher des enfants et des adultes juifs. Avec l'aide du pasteur Pasche, de Léon Leser, juif lui même et qui mit en place un comité de sauvetage des juifs, du résistant Léonce Baron, de son fils et sa belle-fille Pierre-Élie et Odile Nick, médecin établi en Cambrésis où il était facile d'éparpiller les familles dans les fermes de ses patients, le réseau parvint à sauver de nombreux juifs. Le pasteur Nick et ses collaborateurs ont reçu le titre de Justes parmi les Nations.

- Commémoration de septembre 2016 :

La mairie de Lille, la SNCF et Yad Vashem, à la demande de Grégory Célerse, ont organisé une cérémonie commémorative de la rafle et du sauvetage des juifs sur les lieux mêmes de ce qui fut la gare de FivesLille, le 11 septembre 2016. À cette occasion, quelques sauvés ont pu retrouver les familles de leurs sauveteurs. 


\section{Bibliographie : pour en savoir plus}

\section{Sur la rafle et le sauvetage du 11 septembre 1942 :}

- Heddebaut Monique, Sans armes face à la rafle du 11 septembre 1942 (dans la «Zone rattachée » à Bruxelles), article édité à part du $\mathrm{n}^{\circ} 70$ de Tsafon, revue d'études juives du Nord, automne 2015 - hiver 2016.

- Célerse Grégory, Sauvons les enfants, Lille, Les Lumières de Lille, 2016. L'ouvrage rend compte du sauvetage parfois de manière romancée.

\section{Sur la communauté juive de Lens pendant la Guerre :}

- Delmaire Danielle, «Grandeur et misère de la communauté juive de Lens : 1920-1944 », Gauheria, n² 21, juin 1990, p. 65 à 73.

- Un film: Mournaud Carine, Les vies oubliées des Juifs de Lens, Zorn production, 2012, 50 minutes.

- Un ouvrage incontournable: Mariot Nicolas et Zalc Claire, Face à la persécution : 991 Juifs dans la guerre, Paris, Éditions Odile Jacob, 2010, 302 p. Paru en ligne également.

\section{Sur le camp de Malines en Belgique :}

- Schram Laurence, L'antichambre d'Auschwitz - Dossin, Bruxelles, éd. Racine, Fondation Auschwitz, juin 2017.

\section{Sur le sort des juifs du Nord et du Pas-de-Calais pendant la guerre,} Tsafon a publié :

- 1942, l'année tragique des juifs du nord, Tsafon, $\mathrm{n}^{\circ}$ 9-10, été automne 1992, 151 p. Cet ouvrage publie la liste des juifs du Nord et du Pasde-Calais déportés par le camp de Malines, en Belgique, en septembre 1942.

- Déportation et Libération Tsafon $\mathrm{n}^{\circ}$ 21, printemps 1995, 116 p. Édition de documents d'archives, photos, témoignages et liste des juifs du Nord et du Pas-de-Calais déportés par le camp de Malines, en Belgique, 1942 à 1944, autre que septembre 1942.

- Spoliation des Juifs et des Tsiganes dans la Zone rattachée et en Belgique pendant la Seconde Guerre mondiale, Tsafon hors série $\mathrm{n}^{\circ} 5$, novembre 2012. 


\section{Autres études :}

- Delmaire Danielle, «Chrétiens et Juifs dans le Nord et Pas-de-Calais pendant la Seconde Guerre mondiale », Revue du Nord, avril-juin 1978, p. 451 à 456, en collaboration avec Y-M. Hilaire.

- Idem, «Les communautés juives dans la zone interdite pendant l'Occupation ", dans L'occupation en France et en Belgique (19401944), Revue du Nord, nº 2 spécial hors série, 1988, p. 707 à 720.

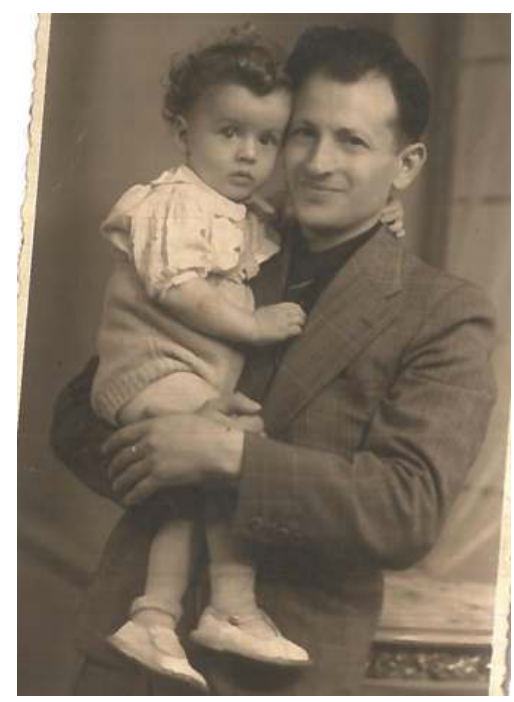

Lejbus Mandelbaum et son fils Daniel en 1942

(archives de la famille Mandelbaum)

Tauba et Lejbus Mandelbaum, réunis en 1946

(archives de la famille Mandelbaum)

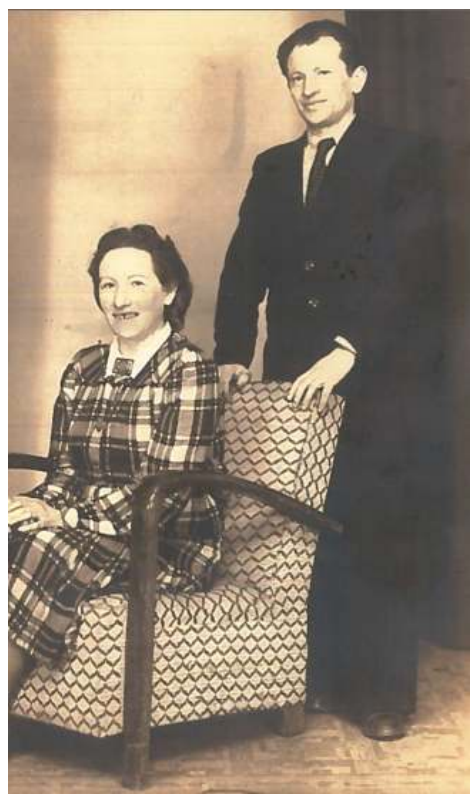

